Gut, 1976, 17, 571-575

\title{
Ascorbic acid deficiency in liver disease
}

\author{
A. D. BEATTIE AND SHEILA SHERLOCK
}

From the Department of Medicine, Royal Free Hospital, London

SUMMARY Leucocyte ascorbic acid (LAA) levels were measured in 138 patients with liver disease. Significantly reduced levels were found in 37 patients with alcoholic liver disease $(\mathrm{P}<0.01)$ and 25 patients with primary biliary cirrhosis $(\mathrm{P}<0.05)$. In the primary biliary cirrhosis patients, cholestyramine therapy was associated with significantly lower levels of the vitamin $(\mathrm{P}<0.05)$. Liver ascorbic acid measured in Menghini needle biopsies in 20 patients was significantly correlated with LAA $(r=0.807$, P $<0.001)$. No significant correlation was found between LAA and haematological indices, conventional liver function tests, or cholesterol levels in any group of patients. Patients with LAA levels below $100 \mathrm{nM} / 10^{8} \mathrm{WBC}$ had significantly higher antipyrine half-lives $($ mean $=28.3 \mathrm{~h})$ than patients with LAA levels above this level (mean $=18.6 \mathrm{~h})(\mathrm{P}<0.05)$. Delayed drug metabolism related to low LAA should be considered when drugs metabolised by the liver are prescribed for patients with alcoholic liver disease or primary biliary cirrhosis.

Ascorbic acid is a water soluble vitamin present in most plant foods especially citrus fruits, potatoes, and green vegetables. Dietary deficiency leads to scurvy and this discovery led to the classic work of Lind (1753) who showed that scurvy could be prevented in sailors by adding citrus fruits to their diet. It is now known that the vitamin is an organic acid whose properties are related to its facility to be oxidised reversibly to dehydroascorbic acid. Its physiological functions are varied and probably not fully worked out. It is generally believed that its oxidation-reduction system plays an important role in biological oxidations and reductions and in cellular respiration. La Du and Zannoni (1961) showed that it is involved in tyrosine metabolism. It is required for the conversion of folic acid to folinic acid. Goldberg (1963) showed that it is required for normal erythropoiesis. Ginter (1973) showed that guinea-pigs with chronic vitamin C deficiency had an impaired conversion of cholesterol to bile acids. Thus the effects of deficiency of the vitamin may be of considerable importance even although signs of scurvy are absent.

Several groups are known to have lower ascorbic acid levels than the general population. It is known that levels fall with age (Andrews and Brook, 1966). Women taking the contraceptive pill have slightly reduced levels (Horwitt et al., 1975). O'Keane et al. (1972) showed that chronic alcoholics have reduced levels. The siderotic Bantu may develop scurvy and

Accepted for publication 4 May 1976 it has been suggested that the iron overload may be a causal factor (Lynch et al., 1967). Since alcoholism and hepatic siderosis are closely related to liver disease the present study was done to find out the prevalence of vitamin $\mathrm{C}$ deficiency in various types of liver disease and to investigate the possible pathophysiological effects in the deficient patients.

\section{Methods}

The 138 patients studied were all inpatients of the University Medical Unit, Royal Free Hospital, London. All suffered from liver disease and the numbers with each diagnosis are shown in Table 1 . The control group consisted of members of staff and patients with non-hepatic disease not known to be related to ascorbic acid deficiency. The age range and sex distribution of the two groups were similar.

Leucocyte ascorbic acid measurement was done on each patient by the method of Denson and Bowers (1961). Ascorbic acid measurement was done on Menghini needle biopsies of the liver in 20 patients using a modification of the method of Denson and Bowers (1961) for leucocyte ascorbic acid. Biopsies of more than $10 \mathrm{mg}$ were considered sufficient. The liver tissue was dried, weighed, and thoroughly homogenised in $1.3 \mathrm{ml} 5 \%$ trichloracetic acid. The homogenate was centrifuged at $3000 \mathrm{rpm}$ for five minutes and $1 \mathrm{ml}$ supernatant incubated for four hours with $0.3 \mathrm{ml} \mathrm{2.2 \% (w/v)} 2.4$ dinitrophenylhydrazine in $10 \mathrm{M} \mathrm{H}_{2} \mathrm{SO}_{4}$ as described in the leucocyte method. The results were expressed 
Table 1 Leucocyte ascorbic acid levels (mean \pm SEM) in 138 patients with liver disease and 28 normal controls

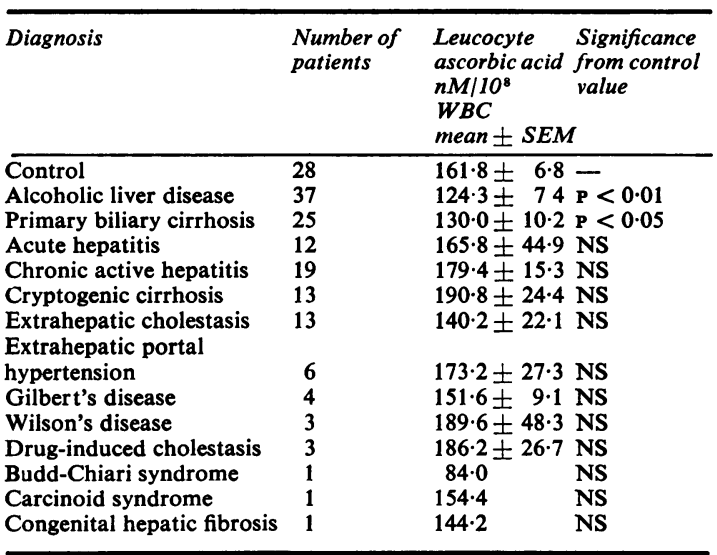

LAA = leucocyte ascorbic acid. Significance is by Student's $t$ test between control and each group of patients.

in $\mathrm{nM}$ ascorbic acid/g liver tissue. Recovery experiments done with 10 to $20 \mu \mathrm{g}$ amounts of normal rat liver gave a mean recovery of $96 \%$ in 12 specimens.

Peripheral blood haemoglobin, mean corpuscular volume, leucocyte count, and platelet count were done on an electronic counter in the haematology department. Serum bilirubin, aspartate transaminase, cholesterol, iron, iron binding capacity, and folate were likewise done by conventional methods in the routine departments.

Plasma antipyrine (phenazone, BPC) half lives were done on 20 patients and four controls. No patient was receiving a drug known to induce liver enzymes and no control subject was receiving any drug at all. Each patient and control received $1.2 \mathrm{~g}$ antipyrine orally and blood samples were taken at approximately $2 \cdot 5$, four, six, eight, 12 , and 24 hours. After separation of the plasma, the samples were stored at $-20^{\circ} \mathrm{C}$ and the antipyrine concentration was measured in batches by the method of Brodie $e t$ al. (1949). The $\mathrm{T}_{\frac{1}{2}}$ of antipyrine disappearance was calculated by regression analysis.

In the in vitro experiment to measure binding of cholestyramine to ascorbic acid, $50 \mathrm{mg}$ cholestyramine were added to $1 \mathrm{ml}$ amounts of aqueous solutions of ascorbic acid in $14.2 \mathrm{M}, 28.4 \mathrm{M}$, and $56.8 \mathrm{M}$ concentrations. The mixture was centrifuged at $3000 \mathrm{rpm}$ for five minutes and the ascorbic acid content of the clear supernatant measured as in the leucocyte method.

The daily dietary intake of ascorbic acid was estimated in the patients with primary biliary cirrhosis according to the dietary equivalent tables in Davidson et al. (1972). The statistical methods used were Student's $t$ test and the coefficient of correlation.

\section{Results}

The mean LAA in the control group was 161.8 $\mathrm{nM} / 10^{8} \mathrm{WBC}$ (SEM $\pm 6 \cdot 8$ ). Overall, the patients with liver disease had a mean of $149.9 \mathrm{nM} / 10^{8}$ WBC $( \pm 7 \cdot 4)$. The mean LAA $( \pm$ SEM) for individual groups of patients is shown in Table 1. Significant reductions in LAA were found in 37 patients with alcoholic liver disease $(124 \cdot 3 \pm 7 \cdot 4$ $\left.\mathrm{nM} / 10^{8} \mathrm{WBC}, \mathrm{P}<0.01\right)$ and 25 patients with primary biliary cirrhosis $\left(130.0 \pm 10.2 \mathrm{nM} / 10^{8}\right.$ WBC, $\mathrm{P}<0.05$ ). No other group differed significantly from control levels.

The 20 patients in whom liver ascorbic acid levels were measured had a mean LAA of $135.0 \mathrm{nM} / 10^{8}$ WBC and a mean liver level of $0.65 \mu \mathrm{M} / \mathrm{g}$ tissue (Figure). A very highly significant correlation was found between the two measurements $(r=0.81$, $\mathbf{P}<0.001)$. The individual results and diagnoses are shown in Table 2.

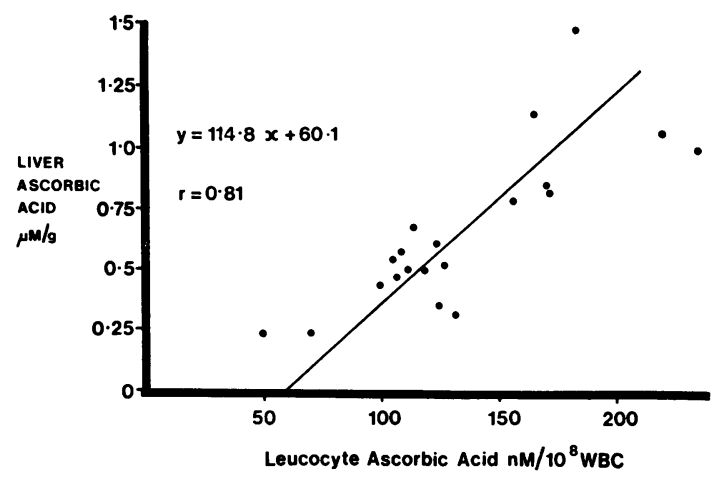

Figure Relationship between $L A A$ and liver ascorbic acid in 20 patients with liver disease. $L A A=$ leucocyte ascorbic acid.

No significant correlation was found between LAA and platelet count ( $\mathrm{n}=86, r=0 \cdot 11)$, serum iron $(\mathrm{n}=22, r=0.05)$, transferrin saturation ( $\mathrm{n}=20, r=0.01$ ), or serum folate $(\mathrm{n}=17, r=$ $0.44)$. A significant negative correlation was present between LAA and mean corpuscular volume ( $\mathrm{n}=95, r=-0.45, \mathrm{P}<0.01$ ). No significant correlation was found between LAA and serum cholesterol in 20 patients with alcoholic liver disease $(r=0.02)$ or in 20 patients with primary biliary cirrhosis $(r=-0.09)$.

Of the 37 patients with alcoholic liver disease, 21 had LAA levels of less than $113 \mathrm{nM} / 10^{8}$ WBC. This 
Table 2 Diagnosis, leucocyte ascorbic acid level, and liver ascorbic acid concentration in 20 patients with liver disease.

\begin{tabular}{lcl}
\hline Diagnosis & $\begin{array}{l}\text { LAA } \\
n M / 10^{8}\end{array}$ & $\begin{array}{l}\text { Liver } \\
\text { ascorbic acid } \\
(\boldsymbol{\mu} M / g)\end{array}$ \\
\hline Alcoholic liver disease & 131.2 & 0.34 \\
Alcoholic liver disease & 49.4 & 0.22 \\
Alcoholic liver disease & 233.9 & 0.99 \\
Alcoholic liver disease & 107.9 & 0.62 \\
Alcoholic liver disease & 127.7 & 0.51 \\
Alcoholic liver disease & 111.8 & 0.49 \\
Alcoholic liver disease & 106.7 & 0.47 \\
Alcoholic liver disease & 69.2 & 0.23 \\
Alcoholic liver disease & 124.9 & 0.34 \\
Alcoholic liver disease & 115.8 & 0.49 \\
Alcoholic liver disease & 156.7 & 0.78 \\
Primary biliary cirrhosis & 123.2 & 0.60 \\
Primary biliary cirrhosis & 105.0 & 0.54 \\
Primary biliary cirrhosis & 113.4 & 0.68 \\
Primary biliary cirrhosis & 98.8 & 0.43 \\
Chronic active hepatitis & 164.1 & 1.13 \\
Chronic active hepatitis & 219.7 & 1.06 \\
Extrahepatic biliary obstruction & 181.1 & 1.48 \\
Extrahepatic biliary obstruction & 170.3 & 0.82 \\
Congestive cardiac failure & 169.8 & 0.84 \\
\hline
\end{tabular}

LAA = leucocyte ascorbic acid.

figure is 2 SD below the control mean and may be regarded as the lower limit of normal. No significant difference was found in serum bilirubin, serum asparatate transaminase, serum cholesterol, serum albumin, or prothrombin time between this group and the 16 patients with normal LAA levels. Eighteen of the 37 patients with alcoholic liver disease had biopsy evidence of hepatic cirrhosis. The mean LAA in these patients was $129.4( \pm 17 \cdot 6)$ $\mathrm{nM} / 10^{8}$ WBC compared with $119.8( \pm 15 \cdot 3) \mathrm{nM} / 10^{8}$ WBC in the 19 patients with non-cirrhotic alcoholic liver disease. This difference is not significant.

LAA levels in the 25 patients with primary biliary cirrhosis did not show a significant correlation with conventional liver function tests. Ten of these patients had levels of less than $113 \mathrm{nM} / 10^{8} \mathrm{WBC}$. The mean plasma caeruloplasmin levels and mean daily dietary intakes of ascorbic acid are shown in Table 3. The patients with low ascorbic acid levels did not have dietary intakes that were significantly different from those with high levels of the vitamin. Nine of the 25 patients with primary biliary cirrhosis were receiving cholestyramine therapy daily in a dose ranging from one to four sachets daily. The mean LAA in the group receiving cholestyramine was $106.2( \pm 11.4) \mathrm{nM} / 10^{8} \mathrm{WBC}$, whereas the patients not receiving this drug had a mean LAA of 143.1 $( \pm 16 \cdot 5) \mathrm{nM} / 10^{8} \mathrm{WBC}$. This is a significant difference $(\mathrm{P}<0.05)$.

When $50 \mathrm{mg}$ cholestyramine were added in vitro to $1 \mathrm{ml}$ amounts of aqueous solutions of ascorbic acid in $14.2 \mathrm{M}, 28.4 \mathrm{M}$, and $56.8 \mathrm{M}$ concentrations respectively, the mean recovery of ascorbic acid was $97 \cdot 3 \%$. This indicates that binding of ascorbic acid in vitro at the $\mathrm{pH}$ of the solution (4.5) did not take place.

Antipyrine half-life in four control subjects was $12.8( \pm 0.9) \mathrm{h}$ and in 20 patients with liver disease was $20.6( \pm 3.8) h$. There was no significant correlation with the LAA level. When Student's $t$ test was performed on groups of patients above and below a given LAA value, however, the trend towards significance increased as the dividing level was reduced. Thus, when the group was divided at 142 $\mathrm{nM} / 10^{8} \mathrm{WBC}$ the $t$ value was $0.88(\mathrm{P}<0.2)$; at 113 $\mathrm{nM} / 10^{8} \mathrm{WBC}$, the $t$ value was $1.89(\mathrm{P}<0.1)$; and at $100 \mathrm{nM} / 10^{8} \mathrm{WBC}$ the $t$ value was $2.40(\mathrm{P}<0.05)$. The mean antipyrine half-life of the four patients with LAA levels less than $100 \mathrm{nM} / 10^{8} \mathrm{WBC}$ was $28 \cdot 3$ $\mathrm{h}$ and of the 16 patients above this level was $18 \cdot 6 \mathrm{~h}$.

\section{Discussion}

The deficiency of vitamin $\mathrm{C}$ in patients with alcoholic liver disease substantiates the work of O'Keane et al. (1972). They showed that alcoholics, whether with or without liver disease, had significantly reduced levels which correlated with the dietary intake of the vitamin. Another possible explanation for the finding of reduced levels is the effect of siderosis. Lynch et al. (1967) showed an accelerated oxidative catabolism of ascorbic acid in siderotic Bantu patients. In the present study there was no correlation between LAA

Table 3 Plasma caeruloplasmin and dietary ascorbic acid intake in patients with primary biliary cirrhosis: $A$ in the group as a whole; $B$ in patients with $L A A<113 \mathrm{nM} / 10^{8} \mathrm{WBC} ; C$ in patients with $\mathrm{LAA}>113 \mathrm{nM} / 10^{8} \mathrm{WBC}$.

\begin{tabular}{|c|c|c|c|c|c|}
\hline Measurement & No of observations & $\begin{array}{l}\text { A } \\
\text { Mean in PBC group } \\
\text { as a whole }\end{array}$ & $\begin{array}{l}\text { B } \\
\text { Mean in patients with } \\
L A A<113 \mathrm{nM} / 10^{8} \\
W B C\end{array}$ & $\begin{array}{l}C \\
\text { Mean in patients with } \\
L A A>113 \mathrm{nM} / 10^{\mathrm{s}} \\
W B C\end{array}$ & $\begin{array}{l}\text { Significance between } \\
B \text { and } C\end{array}$ \\
\hline (mg/day) & 21 & $277 \pm 33$ & $160 \pm 67$ & $252 \pm 37$ & NS \\
\hline
\end{tabular}

LAA = leucocyte ascorbic acid. 
levels and either serum iron or transferrin saturation. Neither of these is as accurate a measurement of siderosis as the serum ferritin or liver iron but it seems unlikely from our results that siderosis is a major cause of ascorbate deficiency in this group of patients.

The low levels of LAA in primary biliary cirrhosis were unexpected and three possible explanations were studied. Firstly, the diet might be deficient. Dietary ascorbic acid was lower in the deficient group but not significantly so, and this led us to look at alternative causes. Secondly, we considered the suggestion of Briggs and Briggs (1972) that women on the contraceptive pill have lower ascorbate levels because of the ascorbate reductase activity of caeruloplasmin (Niedermeier et al., 1967). However, no correlation could be found between LAA levels and caeruloplasmin levels, which suggested that this was not an important cause of the LAA deficiency. The third possibility was that cholestyramine, which nine of the patients were taking because of itching, was binding ascorbate in the gut. This hypothesis appeared to be supported by the finding of significantly lower LAA levels (mean $=106 \cdot 2 \mathrm{nM} / 10^{8}$ WBC) in the group receiving cholestyramine in comparison with the other primary biliary cirrhotic patients (mean 143.1 nM/10 ${ }^{8} \mathrm{WBC}$ ). West and Lloyd (1975) showed that cholestyramine was associated with a reduction in serum folate levels in children and it seems likely that ascorbic acid is similarly affected by the ion exchange resin. The binding could not be confirmed in vitro but this may be due to failure to reproduce the ion exchange conditions which exist in the small intestine.

The hepatic levels of ascorbic acid had a significant correlation with leucocyte levels of the vitamin. This is of some importance, since many of the metabolic actions of ascorbic acid take place in the liver. Since liver levels cannot be easily measured, it is useful to know that they are fairly closely reflected in the peripheral blood.

The possible effects of ascorbic acid deficiency are so varied that only some of them could be considered. Since the conversion of folic acid to folinic acid is facilitated by ascorbic acid, a macrocytic anaemia might be expected to result from deficiency of the vitamin and, indeed, Goldberg (1963) showed that this is so. In our patients there was a significant correlation between LAA and mean corpuscular volume, but the data were not sufficient to show whether this was a separate effect of ascorbic acid deficiency or due to concomitant folate deficiency. Although the folate levels did not correlate in the small number of patients tested, there was a suggestive trend $(r=0.447, \mathrm{P}<0 \cdot 1)$ which probably reflects the partly shared dietary origin of the two substances.

The relationship between serum cholesterol and LAA was first shown by Myasnikov (1950). Since then, there have been several conflicting reports but most authors have found a reduction in cholesterol levels when high doses of ascorbic acid are given to normal subjects (Sokoloff et al., 1967; Ginter et al., 1970). Ginter (1973) showed that conversion of cholesterol to bile salts is delayed in scorbutic guinea pigs and he has suggested that ascorbic acid may be necessary for the formation of bile salt micelles which keep cholesterol dissolved in bile. Thus, ascorbic acid deficiency might enhance gallstone formation. Pedersen (1975) has shown that ascorbic acid in high doses has no effect on biliary lipid composition in normal subjects, but a similar study on patients who are deficient in ascorbic acid might be more rewarding. In the present study there was no correlation between LAA and serum cholesterol in any group but, in view of the effects of cholestasis on serum cholesterol in liver disease, this absence of correlation is not necessarily meaningful.

Delayed drug metabolism has been a known feature of both ascorbic acid deficiency and of impaired liver function for several years. Richards (1941) showed that pentobarbitol sleeping time was prolonged in scorbutic guinea pigs compared with normal controls. Zannoni et al. (1972) showed significant impairment of drug oxidation when hepatic microsomal ascorbic acid had reached $30 \%$ of control values in guinea pigs. Dow et al. (1975) found a significant correlation between LAA and alcohol dehydrogenase in patients with liver disease. Several authors have demonstrated the prolonged elimination of drugs in liver disease (Levi et al., 1968; Mawer et al., 1972; Hvidberg et al., 1974; Klotz et al., 1974). In some of these reports significant correlations have been found with serum albumin levels, while in others this relationship has not been found. Branch et al. (1973) showed prolonged antipyrine half lives in patients with liver disease. In this study we have investigated the possibility that ascorbic acid deficiency is a causal factor in the prolonged drug metabolism of liver disease. From our results it seems that LAA levels below $100 \mathrm{nM} / 10^{8} \mathrm{WBC}$ are likely to be associated with increased drug half life, although prolonged half-life in patients with normal LAA levels suggests that ascorbic acid is only one of several factors involved.

The possibility of ascorbic acid deficiency should be considered in patients with alcoholic liver disease and primary biliary cirrhosis, especially when the diet is poor or when cholestyramine is prescribed. 
When ascorbic acid deficiency is diagnosed, drugs metabolised by hepatic enzymes should be prescribed with caution and possibly in lower dosage until the deficiency is corrected.

We thank Professor B. Billing, Dr N. McIntyre, and Dr S. Barnes for helpful suggestions and Dr J. L. Dormandy who did the caeruloplasmin measurements. A.D.B. was supported by a Medical Research Council Clinical Research Fellowship.

\section{References}

Andrews, J., and Brook, M. (1966). Leucocyte-vitamin-C content and clinical signs in the elderly. Lancet, 1, 13501351.

Branch, R. A., Herbert, M., and Read, A. E. (1973). Determinants of serum antipyrine half-lives in patients with liver disease. Gut, 14, 569-573.

Briggs, M., and Briggs, M. (1972). Vitamin C requirements and oral contraceptives. Nature, 238, 277.

Brodie, B. B., Axelrod, J., Soberman, R., and Levy, B. B. (1949). The estimation of antipyrine in biological materials. Journal of Biological Chemistry, 179, 25-29.

Davidson, S., Passmore, R., and Brock, J. F. (1972). Human Nutrition and Dietetics, 5th edition, p. 136. Churchill Livingstone: Edinburgh.

Denson, K. W., and Bowers, E. F. (1961). The determination of ascorbic acid in white blood cells. Clinical Science, 21, 157-162.

Dow, J., Krasner, N., and Goldberg, A. (1975). Relation between hepatic alcohol dehydrogenase activity and the ascorbic acid in leucocytes of patients with liver disease. Clinical Science and Molecular Medicine, 49, 603-608.

Ginter, E. (1973). Cholesterol: Vitamin C controls its transformation to bile acids. Science, 179, 702-704.

Ginter, E., Kajaba, I., and Nizner, O. (1970). The effect of ascorbic acid on cholesterolemia in healthy subjects with seasonal deficit of vitamin C. Nutrition and Metabolism, 12, 76-86.

Goldberg, A. (1963). The anaemia of scurvy. Quarterly Journal of Medicine, 32, 51-64.

Horwitt, M. K., Harvey, C. C., and Dahm, C. H. (1975). Relationship between levels of blood lipids, vitamins $\mathrm{C}, \mathrm{A}$ and $\mathrm{E}$, serum copper compounds and urinary excretions of tryptophan metabolites in women taking oral contraceptive therapy. American Journal of Clinical Nutrition, 28, 403-412.
Hvidberg, E. F., Andreason, P. B., and Ranek, L. (1974). Plasma half-life of phenylbutazone in patients with impaired liver function. Clinical Pharmacology and Therapeutics, 15, 171-177.

Klotz, U., McHorse, T. S., Wilkinson, G. R., and Schenker, S. (1974). The effect of cirrhosis on the disposition and elimination of meperidine in man. Clinical Pharmacology and Therapeutics, 16, 667-675.

La Du, B. N., and Zannoni, V. G. (1961). The role of ascorbic acid in tyrosine metabolism. Annals of the New York Academy of Science, 92, 175-191.

Levi, A. J., Sherlock, S., and Walker, D. (1968). Phenylbutazone and isoniazid metabolism in patients with liver disease in relation to previous drug therapy. Lancet, 1 , 1275-1279.

Lind, J. (1753). A Treatise of the Scurvy. Reprinted 1953 Edinburgh University Press: Edinburgh.

Lynch, S. R., Seftel, H. C., Torrance, J. D., Charlton, R. W., and Bothwell, T H (1967). Accelerated oxidative catabolism of ascorbic acid in siderotic Bantu. American Journal of Clinical Nutrition, 20, 641-647.

Mawer, G. E., Miller, N. E., and Turnberg, L. A. (1972). Metabolism of amylobarbitone in patients with chronic liver disease. British Journal of Pharmacology, 44, 549-560.

Myasnikov, A. L. (1950). Effect of certain vitamins on cholesterolemia and development of experimental atherosclerosis. Klinicheskaya Meditsina, 28, (No. 2) 3-10.

Niedermeier, W., Lancy, R. P., and Dobson, C. (1967). The mechanism of action of ceruloplasmin in inhibiting ascorbic acid-induced depolymerization of hyaluronic acid. Biochimica et Biophysica Acta, 148, 400-405.

O'Keane, M., Russell, R. I., and Goldberg, A. (1972). Ascorbic acid status of alcoholics. Journal of Alcoholism, 7, 6-11.

Pedersen, L. (1975). Biliary lipids during vitamin C feeding in healthy persons. Scandinavian Journal of Gastroenterology, 10, 311-314.

Richards, R. K., Kueter, K., and Klatt, T. J. (1941). Effect of vitamin $\mathbf{C}$ deficiency on action of different types of barbiturates. Proceedings of the Society for Experimental Biology and Medicine, 48, 403-409.

Sokoloff, B., Hori, M., Saelhof, C., McConnell, B., and Imai, T. (1967). Effect of ascorbic acid on certain blood fat metabolism factors in animals and man. Journal of Nutrition, 91, 107-118.

West, R. J., and Lloyd, J. K. (1975). The effect of cholestyramine on intestinal absorption. Gut, 16, 93-98.

Zannoni, V. G., Flynn, E. J., and Lynch, M. (1972). Ascorbic acid and drug metabolism. Biochemical Pharmacology, 21 1377-1392. 